\title{
Diacronie
}

Studi di Storia Contemporanea

$N^{\circ} 9,1 \mid 2012$

Quando la classe operaia andava in paradiso

\section{Pacifismo, frontismo e autonomia}

Pertini, il Psi e la "fase calda" della guerra fredda (1945-1950)

\section{Gianluca Scroccu}

\section{(2) OpenEdition}

Journals

\section{Edizione digitale}

URL: http://journals.openedition.org/diacronie/2926

DOI: $10.4000 /$ diacronie.2926

ISSN: 2038-0925

Editore

Association culturelle Diacronie

Notizia bibliografica digitale

Gianluca Scroccu, «Pacifismo, frontismo e autonomia », Diacronie [Online], N 9, 1 | 2012, documento 2, online dal 29 janvier 2012, consultato il 10 décembre 2020. URL : http://journals.openedition.org/ diacronie/2926 ; DOI : https://doi.org/10.4000/diacronie.2926 


\section{Diacronie}

\section{Pacifismo, frontismo e autonomia:}

\section{Pertini, il Psi e la "fase calda" della guerra fredda}

\section{(1945-1950)}

\section{Gianluca SCROCCU*}

La storia del Psi negli anni della guerra fredda procede in parallelo con l'evolversi del legame stretto il Pci, in un condizionamento che ne limitò fortemente l'autonomia progettuale sino al 1956. Sandro Pertini, uno dei più importanti dirigenti del partito, cercò inizialmente di mediare tra Nenni e Saragat. Dopo le elezioni del 18 aprile del 1948, però, egli sposò la linea frontista e filosovietica, all'interno della collaborazione stretta coi comunisti. Successivamente egli abbandonò questa linea, peraltro non senza contraddizioni, solo dopo il 1956 e l'avvio della stagione del centro-sinistra e del nuovo corso autonomista.

\section{I socialisti negli anni del frontismo}

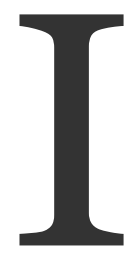

1 tema relativo allimpatto dei condizionamenti delle logiche della guerra fredda sul Psi è diventato un elemento chiave per comprendere le motivazioni e le scelte essenziali del partito guidato da Nenni subito dopo la nascita della Repubblica ${ }^{1}$.

\footnotetext{
1 Cfr. DEGL'INNOCENTI, Maurizio, Sul paradigma socialista o del "terzo partito", in NICOLOSI, Gerardo (a cura di), I partiti politici nell'Italia Repubblicana, Soveria Mannelli, Rubbettino, 2006. SCIROCCO, Giovanni, "La pace è il principio e la fine di ogni cosa": Nenni, il Psi e i percorsi della pace (1948-1969), in CANAVERO, Alfredo, FORMIGONI, Guido, VECCHIO, Giorgio (a cura di), Le sfide della pace. Istituzioni e movimenti intellettuali e politici tra Otto e Novecento, Milano, LED, 2008, p. 145 et seq.
} 
A tal proposito la storiografia è concorde nell'individuare nella scelta filosovietica, perpetuatasi sino al 1956, una delle ragioni dei ritardi strategici del partito e più in generale della sinistra italiana, soprattutto in relazione alle ripercussioni destinate ad avere un peso assai incisivo sino a tempi più recenti. Basti pensare, in tal senso, a quanto accaduto in relazione al mancato sviluppo di un soggetto politico in grado di ripercorrere il cammino portato avanti dalla gran parte della sinistra europea all'interno dell'esperienza del socialismo continentale, o ancora alla contrapposizione fra Pci e Psi dalla metà degli anni Settanta sino al $1992^{2}$.

Come è stato giustamente sottolineato, lo stesso buon risultato alle elezioni del 1946 - dove i socialisti riuscirono ad avere più consensi dei comunisti - poi dilapidato in parte proprio a causa della crescente polarizzazione imposta dal contesto internazionale, può essere visto come un primo momento di svolta3 ${ }^{3}$ L'opzione filosovietica comportò non soltanto la dolorosa scissione di Saragat a Palazzo Barberini ${ }^{4}$, ma di fatto gettò anche sul Psi la spada di Damocle della conventio ad excludendum, ovvero la preclusione dell'accesso al governo per tutte quelle forze politiche che si rifacevano idealmente al mondo sovietico5. Le condizioni per un suo superamento vennero poste solo dopo i fatti del 1956, anche se il percorso non fu agevole e proprio i dubbi sulla fedeltà all'alleanza occidentale ebbero un peso non indifferente nel rallentare l'ingresso del partito nell'area governativa ${ }^{6}$. Tutto questo può contribuire a spiegare perché Simona Colarizi abbia ad esempio molto opportunamente individuato nell'intervallo 1947-1948, il «biennio fatale» destinato a condizionare in

\footnotetext{
2 Cfr. ora ACQUAVIVA, Gennaro, GERVASONI, Marco (a cura di), Socialisti e comunisti negli anni di Craxi, Venezia, Marsilio, 2011.

3 Cfr. MATTERA, Paolo, I socialisti, in GERVASONI, Marco (a cura di), Storia delle sinistre nell'Italia repubblicana, Lungro di Cosenza, Costantino Marco editore, 2011, p. 57.

4 Cfr. DONNO, Michele, Socialisti democratici. Giuseppe Saragat e il Psli (1945-1952), Soveria Mannelli, Rubbettino, 2009.

5 In proposito si rimanda allo studio di GABRIELLI, Gloria, Gli amici americani. I socialisti italiani dalla guerra fredda alle amministrative del 1951-52, Manduria-Bari, Lacaita, 2005. Si veda anche VODOVAR, Christine, Stessa famiglia, diverso approccio. I socialisti italiani di fronte all'America, 1945-1960, in CRAVERI, Piero, QUAGLIARIELLO, Gaetano (a cura di), L'antiamericanismo in Italia $e$ in Europa nel secondo dopoguerra, Soveria Mannelli, Rubbettino, 2004, p. 197.

6 Cfr. NENCIONI, Tommaso, «Tra neutralismo e atlantismo. La politica internazionale del Partito socialista italiano 1956-1966», in Italia contemporanea, 260, settembre 2010, p. 438. Vedi anche BENZONI, Alberto, I socialisti e la politica estera, in BONANNI, Massimo (a cura di), La politica estera della Repubblica italiana, Milano, Comunità, 1967; COLARIZI, Simona, Il partito socialista e la politica di potenza dell'Italia negli anni '5o, in DI NOLFO, Ennio, RAINERO, Romain H., VIGEZZI, Brunello (a cura di), L'Italia e la politica di potenza in Europa (1950-1960), Milano, Marzorati, 1992, pp. 227-232; SCIROCCO, Giovanni, Il PSI dall'atlantismo alla riscoperta dell'Europa (1948-1957), in CRAVERI, Piero, QUAGLIARIELLO, Gaetano (a cura di), Atlantismo ed europeismo, Soveria Mannelli, Rubbettino, 2003, pp. 135204.
} 
maniera risolutiva la storia del socialismo italiano dopo la fine della seconda guerra mondiale7.

La politica estera dei socialisti era in quei frangenti guidata da logiche per lo più politico-strumentali, prive di una loro originalità almeno sino a quando di fatto furono assoggettate ai dogmi provenienti da Mosca. Bisogna inoltre aggiungere i condizionamenti dettati dall'alternarsi delle maggioranze alla guida del partito almeno sino allo stabilizzarsi della svolta frontista di Nenni e Morandi, insieme ai successivi cambiamenti che avrebbero portato all'ingresso nel centro-sinistra ${ }^{8}$.

Relazioni Usa-Urss e questione europea divennero in questo senso i campi privilegiati del dibattito in casa socialista nei primi anni più caldi della guerra fredda 9 . Se nel primo caso la scelta fu netta, almeno nel periodo che va dal 1946-1947 ai fatti di Poznan e di Budapest del 1956, l'approccio al processo di costruzione della Comunità economica europea fu meno rigido, o quantomeno indirizzato a spezzare l'appiattimento dettato dalla logica divisiva della guerra fredda ${ }^{10}$. Anche in questo caso, comunque, non mancarono scontri e lacerazioni, accentuate sicuramente con la firma dei trattati di Roma del 1957 che videro contrapposti soprattutto Lombardi e una parte della sinistra interna ${ }^{11}$. Da questo punto di vista sarebbero diventati centrali sia il Mercato Comune che l'Euratom, anche se il giudizio sul primo sarebbe stato temperato dalla paura che le normative europee legate ad una visione liberoscambista potessero intralciare politiche di pianificazione e non collimare con esigenze di protezione specie per le regioni del Mezzogiorno italiano. Il contrasto tra la posizione dei comunisti, che espressero un voto contrario ai trattati di Roma, e i socialisti, che si astennero, indicò

7 Cfr. COLARIZI, Simona, «I socialisti italiani e l'Internazionale Socialista: 1947-1958», in Mondo contemporaneo, 2/2005, p. 5 .

8 Cfr. DI NOLFO, Ennio, Il socialismo italiano tra $i$ due blocchi, in ISTITUTO SOCIALISTA di STUDI STORICI, Trent'anni di politica socialista (1946-1976), Roma, Mondo Operaio, 1977, pp. 47-48. Per l'ingresso nel centro-sinistra mi permetto di rimandare a SCROCCU, Gianluca, Il partito al bivio. Il Psi dall'opposizione al governo (1953-1963), Roma, Carocci, 2011.

9 Per una storia dei socialisti dopo la caduta del fascismo sino alla Liberazione si rimanda a NERI SERNERI, Simone, Resistenza e democrazia dei partiti. I socialisti nell'Italia del 19431945, Manduria-Bari, Lacaita, 1995.

${ }^{10}$ In generale si veda DECLEVA, Enrico, I socialisti fra unità europea e politica dei blocchi, in COLOMBO, Arturo (a cura di), La Resistenza e l'Europa, Firenze, Le Monnier, 1984, pp. 136181.

${ }^{11}$ In proposito si rimanda a SCIROCCO, Giovanni, Il PSI dall'antiatlantismo alla riscoperta dell'Europa (1948-1957), cit., pag. 195. Vedi anche RAPONE, Leonardo, «I socialisti e l'Europa», in CORTESI, Luigi, PANACCIONE, Andrea (a cura di), Il Ponte: I socialisti e il Novecento: $i$ percorsi, la crisi, LX, 2-3, febbraio-marzo 2004, pp. 173-194; CRUCIANI, Sante, L'Europa delle sinistre. La nascita del Mercato comune europeo attraverso i casi francese e italiano (19551957), Roma, Carocci, 2007; BUFARALE, Luca, «Quale Europa? La sinistra e l'unificazione europea: il caso di Riccardo Lombardi (1943-1957)», in Diacronie. Studi di storia contemporanea, N. $43 \mid 2010$,

URL: < http://www.studistorici.com/2010/10/29/bufarale_numero_4/ > [consultato il 15 gennaio 2012]. 
che la visione del Psi nei confronti dell'economia di mercato e della politica dei blocchi era ormai mutata ${ }^{12}$.

Va detto tuttavia che all'interno di questo tentativo di rottura della logica della guerra fredda fu sicuramente rilevante la riflessione rivolta alle mosse dei Paesi non allineati, attenzione che permise l'aprirsi di spazi di manovra inediti ma al tempo stesso utili per chi ambiva a sganciarsi da una vicinanza troppo stretta all'universo sovietico senza per questo rischiare di essere tacciato di tradimento a favore delle posizioni del blocco occidentale ${ }^{13}$. Naturalmente ciò non poteva procrastinare troppo un giudizio più articolato sul sistema capitalistico statunitense, che se certo si modificò nel cammino verso il governo, grazie anche all'atteggiamento più aperto dell'amministrazione Kennedy nei confronti della partecipazione dei socialisti italiani ad un governo di coalizione con la Democrazia cristiana ${ }^{14}$, non riuscì tuttavia a cancellare una certa diffidenza verso il modello americano, ad esempio da parte del movimento giovanile ${ }^{15}$.

\section{L'unità come fine, l'autonomia come mezzo}

È in questo contesto che si muove un personaggio importante ma sicuramente peculiare della galassia socialista come Sandro Pertini. Uscito vittorioso dalla sua coraggiosa battaglia contro il fascismo, grazie alla quale diventò una delle figure più note e rispettate per il suo eroismo nell'opporsi alla dittatura mussoliniana ${ }^{16}$, egli si apprestava a vivere gli anni della ricostruzione e della Repubblica prima di tutto come militante di un partito a cui aveva deciso di dedicare la sua vita, tanto da sentirlo come una fede. Questo spiega perché in quegli anni, sia come dirigente del Psi che come parlamentare, oltre che come direttore del «Lavoro Nuovo» e dell' «Avanti!»17, Pertini avrebbe preso posizioni che sicuramente possono essere ricondotte al suo particolare

${ }_{12}$ Cfr. PINTO, Carmine, Il riformismo possibile. La grande stagione delle riforme: utopie, speranze, realtà (1945-1965), Soveria Mannelli, Rubbettino, 2008, pp. 126-127.

${ }^{13}$ Cfr. NENCIONI, Tommaso, op. cit., p. 442.

${ }_{14}$ Cfr. GENTILONI SILVERI, Umberto, L'Italia e la nuova frontiera. Stati Uniti e centrosinistra. 1958-1965, Bologna, Il Mulino, 1998. Si veda anche NUTI, Leopoldo, "Socialisti o missili. L'Italia nella politica estera kennediana», in Italia Contemporanea, 204, settembre 1996, pp. 443-470.

15 Sull'atteggiamento del movimento giovanile socialista durante la svolta autonomista, in particolare dopo il 1956, si rimanda a SCROCCU, Gianluca, «Apocalittici o integrati? I giovani della sinistra italiana negli anni del miracolo economico", in Studi e ricerche. Rivista del dipartimento di studi storici, geografici e artistici dell'università di Cagliari, 2, 2009, pp. 187229.

${ }^{16}$ Sulla vita di Pertini dalla nascita al 1945 si rimanda a GANDOLFO, Andrea, Sandro Pertini. Dalla nascita alla resistenza 1896-1945, Roma, Aracne, 2010.

${ }_{17}$ Cfr. PERTINI, Sandro, Anni di guerra fredda. Scritti e discorsi: 1947-1949, Manduria-RomaBari, Lacaita, 2010. 
atteggiamento di devozione verso la causa socialista. Ad una persona che aveva sacrificato anni importanti della sua giovinezza nelle carceri del regime, non si poteva chiedere di non mettere al primo posto della sua esistenza il primato della casa socialista. Supremazia da difendere però contro tutti coloro che avevano mire annessionistiche o prevaricatrici su di esso, ragione che lo avrebbe portato, ad esempio, ad opporsi alla lista unica con i comunisti in occasione delle elezioni del 18 aprile del 1948.

Un elemento decisivo per comprendere le sue scelte negli anni della guerra fredda è quindi, da una parte, il culto dell'unità, che lo avrebbe spinto ad avversare sempre le scissioni, sia quella di Saragat del 1947 che quella della sinistra interna nel 1963-1964, pur non condividendo spesso le scelte della maggioranza alla guida del partito ${ }^{18}$; e dall'altra il vincolo unitario a sinistra che lo induceva a concepire un cammino comune con i militanti comunisti sul piano dell'azione politica e sindacale, seppur con accenti più critici rispetto alle mosse egemoniche dei dirigenti del Pci, specie quando questi intaccavano l'autonomia del suo partito. Sono questi i due punti cardine della sua visione politica, destinati però ad essere sottoposti a forti condizionamenti dalle logiche bipolari che, specie nel secondo caso, resero più partecipi le sue azioni al clima frontista.

La nascita della Repubblica lo convinse infatti che dopo la terribile esperienza della dittatura fascista il movimento operaio avrebbe dovuto diventare il protagonista principale di una nuova Italia, fondata su quei valori che avrebbero sempre rappresentato il suo punto di riferimento: la libertà e la giustizia sociale.

A suo avviso, per realizzare questo obiettivo, i socialisti dovevano stringere un'alleanza più stretta con i comunisti, arrivando a suggellare un patto politico che non poteva non basarsi su un pieno riconoscimento della supremazia politica dell'Unione Sovietica in contrapposizione al blocco occidentale guidato dagli Stati Uniti. Una decisione importante, destinata di fatto a segnare in maniera radicale la storia della sinistra italiana ma soprattutto una scelta disastrosa per il Psi in quanto fu un elemento che l'avrebbe condannato ad una condizione perpetua di minoranza all'interno della sinistra italiana a tutto vantaggio del Pci. Pertini non poté che sposare questa visione:

18 Pertini fu sempre persuaso che la critica fosse legittima purché si svolgesse all'interno degli organismi dirigenti e non diventasse occasione sfruttabile dalla propaganda avversaria; la minoranza, a suo giudizio, doveva essere sempre rispettata e avere piena cittadinanza senza per questo pensare di risolvere la sua condizione di inferiorità tramite distacchi traumatici. Per un'analisi più ampia dell'atteggiamento tenuto da Pertini rispetto alle varie dinamiche interne dei socialisti italiani dal 1945 sino al varo del primo governo Moro nel dicembre $1963 \mathrm{mi}$ permetto di rimandare a SCROCCU, Gianluca, Sandro Pertini e il Psi dalla Liberazione al centro-sinistra, Manduria-Bari-Roma, Lacaita, 2008. 
del resto, in un uomo con la sua storia personale, il richiamo all'antifascismo e alla Resistenza, quest'ultima concepita soprattutto come unità di tutte le forze popolari, avrebbe inciso sempre in profondità sul suo agire politico.

Esemplificativo in tal senso il suo intervento al Senato del 14 luglio del 1948, subito dopo l'attentato a Togliatti, quando ribadì la sua piena solidarietà «al partito comunista che per venti anni si è battuto contro il fascismo e per le libertà democratiche», sottolineando, al contrario, di non aver incontrato durante gli anni del carcere imposti dal regime mussoliniano gli uomini che sedevano alla sua destra nell'emiciclo di Palazzo Madama ${ }^{19}$.

\section{Tra autonomia e frontismo: Pertini tra vincolo di classe e impegno per l'autonomia del partito}

È bene premettere che già alla prima riunione dei socialisti dopo la Liberazione, il Consiglio Nazionale tenuto a Roma dal 29 luglio al $1^{\circ}$ agosto 1945, erano emerse le prime divaricazioni che testimoniavano forti tensioni interne per quanto riguarda l' identità del partito e i suoi obiettivi politici ${ }^{20}$. Due erano le tendenze che si confrontarono: da una parte il gruppo raccolto attorno a Nenni il quale, prendendo spunto dalle speranze suscitate dal governo Parri e dalle aspettative di profondo cambiamento delle strutture politiche italiane, riteneva che il partito dovesse impegnarsi soprattutto sul problema della Costituente e della costruzione dell'alleanza unitaria con il Pci; dall'altra, invece, il gruppo guidato da Saragat e Silone, che sosteneva la necessità dell'autonomia socialista, iscrivendola nel solco della funzione mediatrice della famiglia del socialismo europeo ${ }^{21}$, affermatasi in Inghilterra con la vittoria del Labour Party del luglio $1945^{22}$.

Pertini, che aveva aderito alla mozione di Nenni come primo firmatario, intervenne durante l'assise per ribadire la necessità dell'unità del movimento operaio, criticando le posizioni contrarie di Saragat all'unione organica con i comunisti che a suo avviso erano da rifiutare perché bisognava respingere l'intento di rinchiudersi in uno

\footnotetext{
19 Cfr. PERTINI, Sandro, Discorsi parlamentari 1945-1976, Roma-Bari, Laterza, 2006, p. 34.

${ }^{20}$ Cfr. TADDEI, Francesca, Il socialismo italiano del dopoguerra: correnti ideologiche e scelte politiche (1943-1947), Milano, Franco Angeli, 1984, p. 141.

${ }^{21}$ Cfr. CAFAGNA, Luciano, Una strana disfatta. La parabola dell'autonomismo socialista, Venezia, Marsilio, 1996, p. 20.

${ }^{22}$ Le divaricazioni presenti allinterno del partito in questa fase sono state ben analizzate, specie per quanto riguarda la corrente socialdemocratica, in STRINATI, Valerio, Politica e cultura nel PSI, Napoli, Liguori, 1980, pp. 29-30.
} 
splendido isolamento [...] dalla classe operaia. Saragat dice che la nostra è l'ora del socialismo e non l'ora dell'unità proletaria. Questo significa rinviare un problema che non si vuole risolvere, chiudersi nella torre d'avorio del nostro partito come si chiuse il partito comunista che, pur stringendo alleanze con altri partiti, nel passato pensava di essere depositario della verità rivelata e di rappresentare esso solo l’idea socialista. ${ }^{23}$

La sua paura principale era in sostanza che si verificasse una situazione di instabilità e di contrasti insanabili tra i partiti dei lavoratori paragonabile a quella del 1922 che aveva permesso l'avvento del fascismo ${ }^{24}$. Un tema, questo, destinato a tornare sovente nella retorica pertiniana di quegli anni.

In quella fase il mito dell'unità dei lavoratori agiva in lui come potente richiamo politico e culturale. In quest'ottica egli auspicava un partito unico della sinistra, anche se il clima di euforia post-Liberazione non poteva nascondere le insidie celate dietro un simile progetto ${ }^{25}$. Pertini era infatti conscio dell'impossibilità che un partito del genere vedesse subito la luce in quanto il processo di unificazione sarebbe dovuto essere graduale, partecipativo e regolato da due principi inderogabili: la democrazia interna e l'assoluta indipendenza da ogni interferenza di forze esterne. Un'esternazione che palesava evidentemente un certo imbarazzo se non una vera e propria percezione deformata della realtà, visto che lo scenario bipolare non avrebbe certo consentito una strategia in tal senso.

Bisogna però sottolineare che nei mesi successivi, soprattutto in previsione del congresso del partito dell'aprile del 1946, le sue posizioni fusioniste iniziarono ad essere meno granitiche. Egli infatti aderì alla corrente di «Iniziativa Socialista», una proposta di mediazione tra l'ala fusionista e le posizioni degli uomini vicini a Saragat, avente una concezione assai più complessa su questioni dirimenti, prima fra tutte il fronte unico insieme ai comunisti concepito come qualcosa che «non dovesse essere una catena che ci legasse a tal punto da portarci nel pozzo, se mai volevano andarci loro». La sua adesione a «Iniziativa socialista» era stata dettata dal fatto che questa piattaforma aveva avuto il merito di aggiungere chiarezza e far diminuire la tensione nelle varie

${ }^{23}$ Cfr. PERTINI, Sandro, «Al Consiglio Nazionale», ora in PERTINI, Sandro, Scritti e discorsi di Sandro Pertini, vol. I, 1926-1978, Roma, Presidenza del Consiglio dei Ministri, Dipartimento per l'Informazione e l'Editoria, 1992, p. 51.

24 Ibidem.

${ }_{25}$ Cfr. PERTINI, Sandro, «La democrazia», in L'epoca, 8 agosto 1945, poi ripubblicato sul Lavoro nuovo, 15 agosto 1945, ora in ID., Scritti e discorsi di Sandro Pertini, cit., p. 54. 
componenti del partito, superando l'equivoca e sfumata situazione interna manifestatasi nelle settimane precedenti.

In un momento in cui non si poteva realizzare una rivoluzione proletaria, Pertini temeva che la classe operaia potesse rimanere esclusa dalla vita politica, ragione per la quale la funzione del Partito socialista avrebbe dovuto essere quella di agire da

trait-d'union fra la classe operaia e le altri correnti politiche. Il partito socialista, infatti, è noto come un partito sinceramente democratico ed ha un contenuto ideologico identico a quello del partito comunista. Quindi per questa sua somiglianza con i partiti democratici e per la sua identità di pensiero e finalità con il partito comunista esso è il più indicato a fare da ponte fra la classe operaia controllata dal PCI e le altri correnti politiche. ${ }^{26}$

Il Partito socialista doveva ricoprire a suo avviso questo ruolo fondamentale di mediazione ed unione, che non sarebbe stato capace di esplicitare se si fosse fuso con $\mathrm{i}$ comunisti. Quindi, per Pertini, il no alla fusione era motivato non sulla base di preclusioni di carattere ideologico-programmatico, come per Saragat, ma dalla convinzione che sarebbe stata un limite al reale ruolo che avrebbero dovuto giocare $\mathrm{i}$ socialisti nello scacchiere politico italiano, immuni com'erano da condizionamenti esterni, mentre «i democristiani erano un partito confessionale legato al Vaticano, i comunisti all'Urss» ${ }^{27}$.

$\grave{E}$ bene ricordare che l'assise nel capoluogo toscano sarebbe stata la prima ad essere pesantemente condizionata dagli sviluppi della contrapposizione bipolare. Si pensi solo alle conseguenze dirompenti del discorso pronunciato da Churchill all'Università di Fulton nel mese precedente, intervento con il quale l'ex premier inglese aveva denunciato e duramente criticato la politica sovietica e il suo voler estendere «una cortina di ferro» nel cuore dell'Europa ${ }^{28}$.

I protagonisti del congresso furono Nenni e Saragat e il loro confronto fu il primo round di uno scontro destinato a ripetersi nei mesi successivi. Nenni aveva assunto sin dal principio una posizione fortemente unitaria. A suo avviso i partiti di sinistra avevano subito dure sconfitte ogni qual volta si erano mostrati tra di loro divisi e ostili; socialisti e comunisti non potevano prescindere dalla loro comune origine di classe e

${ }^{26}$ Ibidem, p. 71.

27 Ibidem.

${ }_{28}$ Sulle conseguenze innescate dal discorso del premier britannico si rimanda a ROMERO, Federico, Storia della guerra fredda. L'ultimo conflitto per l'Europa, Torino, Einaudi, 2009, p. 43; PINZANI, Carlo, Il bambino e l'acqua sporca. La guerra fredda rivisitata, Milano, Le Monnier-Mondadori Education, 2011, p. 43. 
dal richiamo al marxismo e pertanto le finalità reciproche non potevano divergere sebbene fossero organizzati in due soggetti distinti. Saragat, viceversa, ebbe buon gioco nell'accusare il suo antagonista di volersi legare in un vincolo troppo stretto coi comunisti, inseguendo così il modello di un partito totalitario che si ispirava ad un'esperienza fortemente antidemocratica come quella sovietica. Il futuro leader socialdemocratico criticò anche Basso cui imputò il fatto di richiamarsi ad un marxismo rigidamente classista, troppo simile al modello leninista, quando c'era invece bisogno dell'affermarsi di un socialismo democratico e umanitario ${ }^{29}$.

Nel suo intervento dalla tribuna congressuale, specialmente nel punto riguardante la politica estera, Pertini fece una perorazione a favore dell'internazionalismo socialista, ritenendo che il socialismo europeo potesse affrontare meglio le sfide per il miglioramento delle condizioni delle classi popolari soltanto con una strategia comune $^{30}$. Un'Internazionale Socialista dotata di una grande autorità, a suo avviso, avrebbe liberato la Russia dal pericolo di essere aggredita dagli stati imperialisti, aiutandola a riprendere il cammino di una più autentica democrazia. Un giudizio, evidentemente, che non teneva conto di come oramai la situazione avesse assunto i contorni di una guerra ideologica assai radicalizzata che non ammetteva posizioni sfumate, ragione per la quale posizioni come la sua apparivano ancora non pienamente definite.

E questo si vide bene quando, in risposta alle dichiarazioni di Saragat che, in un colloquio con il segretario di Stato americano Byrnes avvenuto durante la conferenza di Parigi, aveva sollecitato un impegno statunitense per porre un argine alla politica filosovietica del Pci, fu proprio Pertini, per conto di tutto il Psi, a difendere Saragat dalle colonne dell'«Avanti!» dalle dure critiche rivoltegli da Togliattiji ${ }^{1}$. Egli, come si è già detto, aveva intuito che il partito sarebbe stato ridimensionato da un'eventuale scissione della sua componente riformista, e che questo avrebbe avvantaggiato

29 Sul congresso si veda l'analisi di MATTERA, Paolo, Il Partito inquieto. Organizzazione, passioni e politica dei socialisti italiani dalla Resistenza al miracolo economico, Roma, Carocci, 2004, pp. 100-101.

${ }^{30}$ Cfr. PERTINI, Sandro, «Al Congresso di Firenze», Italia socialista, 5 maggio 1946, ora in ID., Scritti e discorsi di Sandro Pertini, cit., p. 79: «Guai, o compagno Nenni, se i partiti socialisti d'Europa dovessero rimanere staccati gli uni dagli altri; guai se non sentissero questo i socialisti d'Europa, che si è socialisti veri se anche e soprattutto internazionalisti».

${ }^{31}$ Cfr. PERTINI, Sandro, «Il terzo gode», in Avanti!, 21 settembre 1946. Tutta la vicenda è ben ricostruita in LUCIA SERGIO, Maria Luisa, De Gasperi e la «questione socialista». L'anticomunismo democratico e l'alternativa riformista, Soveria Mannelli, Rubbettino, 2004, p. 108. 
soprattutto il Pci dal punto di vista elettorale ${ }^{32}$. In questo senso lo scenario dell'unità a sinistra non poteva concretizzarsi, a suo parere, a prezzo della solidità interna del partito. Una prospettiva ambiziosa, quella di Pertini, ma destinata a rivelare tutta la sua fragilità di fronte all'evolversi inesorabile degli eventi, che peraltro avrebbero modificato in profondità anche la sua posizione.

\title{
4. La rottura del fragile equilibrio: l'esclusione delle sinistre dal governo
}

Il IV governo De Gasperi segnò una svolta radicale nella politica della neonata Repubblica, essendo nato come conseguenza della rottura della unità antifascista che aveva rappresentato il collante tra i partiti italiani33. In un articolo pubblicato sul «Lavoro nuovo» il 29 maggio 1947, Pertini espresse tutta la sua preoccupazione verso una soluzione che riteneva ingiusta e antidemocratica, dettata soprattutto dalla diversa concezione e dai condizionamenti degli Stati Uniti nelle vicende interne della politica italiana. A suo avviso, infatti, la sinistra era consapevole sia della riconoscenza che il popolo italiano aveva nei confronti degli USA per il loro intervento contro il regime fascista, sia del peso che gli aiuti d'oltreoceano avrebbero potuto rappresentare per la ricostruzione economica del Paese. Tutto questo, però, non poteva avvenire a prezzo di sacrificare l’indipendenza e l'autonomia nazionale:

\begin{abstract}
Quest'aiuto non potrà essere accettato che con riconoscenza, se esso ci sarà offerto per favorire la nostra ripresa economica e di conseguenza la nostra capacità d'acquisto e per far sì che il mercato europeo possa al più presto assorbire gran parte della produzione americana, onde sia evitato agli Stati Uniti di cadere in un grave crisi. Se, invece, l'aiuto dovesse esserci offerto con l'intenzione di far entrare l'Italia nell'orbita del mondo occidentale, noi non potremmo accettarlo. 34
\end{abstract}

\footnotetext{
${ }^{22}$ Cfr. SABBATUCCI, Giovanni, Il riformismo impossibile. Storie del socialismo italiano, RomaBari, Laterza, 1991, pp. 80-81.

33 Cfr. SCOPPOLA, Pietro, La Repubblica dei partiti. Profilo storico della democrazia in Italia (1945-1990), Bologna, Il Mulino, 1991, pp. 144-148; GIOVAGNOLI, Agostino, Il partito italiano. La Democrazia Cristiana dal 1942 al 1994, Roma-Bari, Laterza, 1996, pp. 34 et seq.; LEPRE, Aurelio, Storia della prima Repubblica. L'Italia dal 1943 al 1998, Bologna, Il Mulino, 1999, pp. 87-92; MALGERI, Francesco, La stagione del centrismo. Politica e società nell'Italia del secondo dopoguerra (1945-1960), Soveria Mannelli, Rubbettino, 2002, pp. 51-53; FORMIGONI, Guido, "De Gasperi e la crisi politica italiana del maggio 1947», in Ricerche di storia politica, 6, 3/2003; CRAVERI, Piero, De Gasperi, Bologna, Il Mulino, 2006, pp. 295-302. 34 Cfr. PERTINI, Sandro, «In difesa della patria», in Lavoro nuovo, 5 agosto 1947, ora in ID., Scritti e discorsi di Sandro Pertini, cit., p. 138.
} 
Il progetto di isolare le sinistre dal governo rappresentava ai suoi occhi un grave errore dei democristiani, in un momento in cui la classe operaia aveva acquisito il diritto a veder realizzate le proprie rivendicazioni e a partecipare alla ricostruzione della democrazia nel paese 35 .

Da quel momento si sarebbe aperta una fase incandescente della politica italiana che avrebbe visto i mesi successivi segnati da un'intensa quanto drammatica asprezza: si avvicinava infatti la resa dei conti delle elezioni del 18 aprile 1948.

Lo scenario in cui si inseriscono questi eventi fece da sfondo al XXVI congresso nazionale del Psi tenutosi a Roma tra il 19 e il 22 gennaio 1948, dominato e vinto dalla forza oratoria di Nenni che con il suo intervento del 21 gennaio persuase della necessità dell'alleanza elettorale con i comunisti molti delegati che avevano manifestato perplessità durante la fase precongressuale 36 .

Pertini si mosse perfettamente all'interno dei confini di questa proposta. Dal punto di vista della politica internazionale riconobbe al popolo americano la gratitudine per gli aiuti all'Italia attraverso il Piano Marshall, ma ribadì che questo non doveva essere confuso con la politica «imperialistica» di Truman la quale mirava ad asservire sul piano economico e politico le nazioni europee. Diverso era invece l'atteggiamento da tenere nei confronti dell'Unione Sovietica:

È un fatto che i paesi che sono sotto l'influenza dell'Unione Sovietica vanno realizzando profonde riforme di struttura. Si tratta di assecondare il fatto storico che si è creato nel mondo con la rivoluzione sovietica e di imprimere ad esso le nostre caratteristiche, quelle del partito socialista. ${ }^{37}$

$\mathrm{Fu}$ un intervento in cui predominò un linguaggio già ispirato alle logiche frontiste: duri attacchi alla Dc, accusata di spingere la classe operaia nell'illegalità; sferzanti critiche all'azione di Saragat e del repubblicano Randolfo Pacciardi, incolpati di tradimento ai danni dei lavoratori e di essersi legati alle forze della reazione ${ }^{38}$. Ritornava inoltre il leitmotiv circa la necessità che le clausole di accettazione del sistema di aiuti del Piano Marshall non si trasformassero in una perdita della propria

35 Cfr. PERTINI, Sandro, «Punto e a capo», Lavoro nuovo, 5 agosto 1947, ora in ID., Anni di guerra fredda. Scritti e discorsi: 1947-1949, cit., p. 70.

${ }_{36}$ Sul congresso si veda l'analisi di MATTERA, Paolo, Il Partito inquieto, cit., pp. 136-137.

37 Cfr. PERTINI, Sandro, «Intervento al XXVI Congresso», Avanti!, 21 gennaio 1948, ora in ID., Scritti e Discorsi di Sandro Pertini, cit., p. 167.

${ }^{38}$ Ibidem, pp. 167-168. 
indipendenza relativa alle scelte della politica nazionale39. Un discorso, questo, che Pertini riprese anche in occasione del dibattito alla Costituente sulla ratifica del Trattato di Pace40. Quest'ultimo, come è noto, venne aspramente criticato dai socialisti che infatti non avrebbero partecipato alla votazione parlamentare relativa alla sua ratifica; toni differenti sarebbero stati usati, invece, quando fu l'Urss a ratificare il trattato, momento esaltato dallo stesso Pertini come evento fondamentale e viatico per l'ammissione dell'Italia all'Onu41.

Tanto più rilevante appare in questo contesto la data fatidica del 18 aprile 1948, giornata percepita come un momento di svolta epocale dai principali partiti ${ }^{42}$. In quelle settimane l'azione politica di Pertini si allineò totalmente con gli intendimenti della politica frontista, mentre le sue sfumature e le sue posizioni tutte ancorate alla ricerca dell'autonomia e dell'unità interna vennero accantonate. Non era tempo per richiamarsi all'unità socialista: serviva una retorica che fosse duramente critica nei confronti del governo, di De Gasperi, della politica americana43, con toni spesso sprezzanti contro i vecchi compagni scissionisti socialdemocratici. Nella propaganda, poi, l'esigenza del fronte unico esigeva richiami alla più stretta collaborazione con i comunisti, con una comunanza che si manifestava soprattutto durante i comizi quando si dovevano tessere lodi sperticate dell'Unione Sovietica e delle conquiste del «socialismo realizzato» 44 .

Pertini era convinto che una vittoria del Fronte nella consultazione elettorale avrebbe aperto le porte alla fondazione di un nuovo stato pronto ad inserirsi in piena autonomia nello scenario politico internazionale e capace di emancipare e condurre al

39 Cfr. PERTINI Sandro, «Per la ricostruzione dell'Europa e per la pace nel mondo», in Lavoro Nuovo, 10 luglio 1947, ora in ID., Anni di guerra fredda. Scritti e discorsi: 1947-1949, cit., pp. 87-88.

40 Cfr. PERTINI Sandro, «In difesa della patria», in Lavoro Nuovo, 5 agosto 1947, ora in ID., Anni di guerra fredda. Scritti e discorsi: 1947-1949, cit., pp. 91-93.

${ }^{41}$ Cfr. PERTINI Sandro, «La strada della pace», in Lavoro Nuovo, 2 settembre 1947, ora in ID., Anni di guerra fredda. Scritti e discorsi: 1947-1949, cit., p. 96.

${ }^{42}$ Sulla campagna elettorale del 18 aprile cfr. LUPO, Salvatore, Partito e antipartito. Una storia politica della prima Repubblica (1946-78), Roma, Donzelli, 2004, pp. 49-59; GELSOMINI, Elena, Le campagne elettorali della prima repubblica, Manduria-Roma-Bari, Lacaita, 2009, pp. 13-105; RIDOLFI, Maurizio, Storia politica dell'Italia repubblicana, Milano, Bruno Mondadori, 2010. Per un'analisi su Dc e Pci si rimanda alla nuova edizione aggiornata di VENTRONE, Angelo, La cittadinanza repubblicana. Come cattolici e comunisti italiani hanno costruito la democrazia italiana (1943-1948), Bologna, Il Mulino, 2008; in particolare sulla campagna del 1948 si vedano le pp. 253-272.

43 Cfr. PERTINI, Sandro, «Non pensano alla patria», in Lavoro Nuovo, 8 gennaio 1948, ora in ID., Anni di guerra fredda. Scritti e discorsi: 1947-1949, cit., pp. 159-162. Su questi temi si rimanda ai saggi presenti in CRAVERI, Piero, QUAGLIARIELLO, Gaetano (a cura di), L'antiamericanismo in Italia e in Europa nel secondo dopoguerra, cit.

${ }_{44}$ Cfr. MATTERA, Paolo, Il partito inquieto, cit., pp. 141-143. 
potere la classe lavoratrice45. Nei suoi comizi il riferimento alla divisione tra Usa e Urss fu sempre molto presente: il suo refrain preferito era il richiamo alla solidarietà internazionale tra i lavoratori, compresi quelli statunitensi, cui però si accompagnava sempre un cenno al non dover barattare gli aiuti economici con il sacrificio della propria indipendenza nazionale e l'asservimento alle logiche del dominio di una superpotenza, che naturalmente era rappresentata dal governo di Washington ${ }^{46}$. In questo senso furono molto dure le sue critiche al Piano Marshall sino agli ultimi giorni della campagna elettorale, come testimoniato anche dalle segnalazioni delle questure ${ }^{47}$.

I dati elettorali si rivelarono però impietosi, assegnando una vittoria schiacciante alla Democrazia Cristiana. Pertini commentò così la sconfitta sul «Lavoro nuovo» e poi sull'«Avanti!», cercando, per quanto gli era possibile, di non far trapelare troppo la delusione:

Stolto è sostare per recriminare vanamente. Bisogna rimanere in piedi e continuare a lottare. [...] Certo però è questo: che sarà raggiunta la meta per cui ci battiamo. Tutti oramai avvertono che la storia d'Europa si trova ad una svolta decisiva e i primi ad avvertirlo sono i nostri avversari e questo spiega il loro accanimento nel combatterci. Ma nessuno potrà fermare la ruota della Storia e quello che sembra sogno oggi sarà realtà domani.48

\section{Dopo la bufera. Pertini e il Psi negli anni del frontismo}

Come si è già sottolineato, la débâcle del 18 aprile lasciò la sinistra in un grave stato di confusione politica, perché segnò la sconfitta della strategia che aveva ispirato l'unità d'azione tra i due partiti49.

Quella disfatta aveva comunque lasciato i suoi segni soprattutto sul Psi e in particolare sull'universo dei militanti. La subalternità dei socialisti verso i comunisti contrastava però con la persistenza di alcuni elementi portanti della storia del partito, a

45 Cfr. PERTINI, Sandro, «...E il fronte avanza», Lavoro nuovo, 4 marzo 1948, ora in ID., Scritti e Discorsi di Sandro Pertini, cit., p. 175.

${ }^{46}$ Cfr. PERTINI, Sandro «Se la libertà fosse in pericolo...», Avanti!, 15 aprile 1948, ora in ID., Scritti e Discorsi di Sandro Pertini, cit., p. 175. Una sintesi in "Grande discorso di Pertini a Roma», in Lavoro Nuovo, 15 aprile 1948, ora in ID., Anni di guerra fredda. Scritti e discorsi: 1947-1949, cit., p. 185.

47 Cfr. SCIROCCO, Giovanni, Politique d'abord, cit., p. 29.

48 Cfr. PERTINI, Sandro, «Avanti compagni», Lavoro nuovo, 22 aprile 1948, ora in ID., Scritti e Discorsi di Sandro Pertini, cit., p. 177.

49 Cfr. DEGL'INNOCENTI, Maurizio, Storia del Psi, vol. III, Dal dopoguerra a oggi, Roma-Bari, Laterza, 1993, p. 111. 
partire dalla vivace dialettica interna e dai diversi posizionamenti che ne scaturivano ${ }^{50}$. I maggiori responsabili di quella situazione apparivano Nenni e Morandi, vale a dire i dirigenti che con maggiore forza avevano spinto per la costituzione del fronte comune con il Pci.

Il XXVII congresso, tenutosi a Genova tra il 27 giugno e il 1 luglio 1948, rappresentò in quel frangente un momento di svolta, con un arretramento delle tendenze più legate al frontismo e una ripresa dell'iniziativa politica di quei dirigenti più vicini a posizioni che si potrebbero definire centriste 51 .

In questa situazione pareva naturale che un uomo come Pertini, che aveva svolto benissimo e disciplinatamente il proprio ruolo nella campagna elettorale, partendo però da una contrarietà di fondo nei confronti delle liste unificate, sentisse l'esigenza di appoggiare una linea politica diversa da quella espressa dal gruppo guidato da Basso e Nenni. Tutto questo può contribuire a spiegare perché in vista del congresso Pertini sostenne il documento di «Riscossa socialista» 52 , insieme a dirigenti di spicco come Jacometti, Lombardi53, Foa e Santi. Una mozione, quella centrista, che aveva come obiettivo la continuazione dell'alleanza con il Pci ma in una condizione di maggiore autonomia e libertà di scelta politica, espressa ad esempio dallo scioglimento dell'alleanza frontista e dalla ricerca per il partito di una posizione di equidistanza tra i due blocchi.

L'intervento di Pertini dalla tribuna congressuale spiazzò però i suoi compagni di mozione, perché di fatto costituì un richiamo ai temi della politica di unità d'azione con i comunisti ribaltando così l'essenza della piattaforma del documento54.

Certamente è bene rilevare come, a parziale giustificazione delle parole di Pertini, durante il cammino congressuale la mozione non si fosse certo caratterizzata per la compattezza dei suoi aderenti55. Resta il fatto che la reazione alle sue parole, indubbiamente un cambiamento di atteggiamento inaspettato, non si fece attendere: ci fu chi mise in evidenza che il suo intervento aveva di fatto segnato la spaccatura di

${ }^{\circ}$ Cfr. SALVADORI, Massimo Luigi, La Sinistra nella storia italiana, Roma-Bari, Laterza, 2001, p. 105.

${ }^{51}$ Si rimanda in proposito a MATTERA, Paolo, Il Partito inquieto, cit., p. 152.

${ }^{2}$ Per la ricostruzione accurata dei temi principali del congresso cfr. SCIROCCO, Giovanni, Politique d'abord, cit., pp. 32-33.

53 Cfr. TORTORETO, Emanuele, Riccardo Lombardi e le relazioni internazionali dalla Resistenza al 1957, in RICCIARDI, Andrea, SCIROCCO, Giovanni (a cura di), Per una società diversamente ricca. Scritti in onore di Riccardo Lombardi, Roma, Edizioni di Storia e Letteratura, 2004, pp. 45-53.

54 Cfr. SCIROCCO, Giovanni, Politique d'abord, cit., p. 35.

55 Cfr. DE MARTINO, Francesco, Intervista sulla sinistra italiana, Roma-Bari, Laterza, 1998, p. 40. 
«Riscossa», chi invece ne apprezzò la capacità di rimettere al centro la questione dell'unità della classe operaia e della sinistra.

Per la maggior parte degli aderenti al documento, comunque, le sue argomentazioni provocarono molta amarezza, venendo viste come una retromarcia foriera di ulteriori divisioni e smarrimenti dopo la dura sconfitta elettorale. Emblematiche furono, in questo senso, le parole di Vittorio Foa:

Dopo il 18 aprile un gruppo di socialisti contrari alla scelta elettorale frontista portarono la questione al congresso. [...] Proponemmo a Sandro Pertini, prestigioso uomo della resistenza, di essere il nostro capofila. Non era frontista e accettò. La linea era: stretta collaborazione coi comunisti sul piano politico e sindacale, totale indipendenza sul piano sociale e sindacale, totale indipendenza sul piano politico, equidistanza o non allineamento fra America e Russia. Andammo a congresso a Genova. L'usanza congressuale era che i capofila dei diversi schieramenti presentassero con un discorso le loro mozioni, che poi dovevano essere discusse e votate. La mozione filocomunista fu presentata da Nenni, la mozione di orientamento socialdemocratico fu presentata da Giuseppe Romita, che era stato ministro degli Interni nel governo di coalizione nel momento delicato del passaggio alla repubblica. Poi toccò a Pertini il quale, senza averci detto niente prima, invece della nostra posizione illustrò quella di Nenni. Noi restammo tutti a bocca aperta..$^{6}$

Da allora le sue prese di posizione ispirate ad un filo-frontismo sempre più marcato divennero più numerose, spesso con sottolineature degli intenti egemonici delle potenze occidentali contro i tentativi sovietici di costruire un mondo regolato dalla pace e dal mutuo rispetto fra i due grandi blocchi.

Tutto questo mentre il gruppo dirigente alla guida del partito, con a capo Jacometti e Lombardi, faticava a reggere con mano ferma il nuovo corso del partito sia per le difficoltà legate ad una vittoria che aveva assicurato solo una maggioranza relativa dei consensi, sia per l'azione di condizionamento esterno del Pci che con il sistema della doppia tessera forniva un valido aiuto alla sinistra interna57. Tale situazione si risolse in un nuovo congresso che nelle intenzioni di Nenni e Morandi avrebbe dovuto sancire la fine della segreteria centrista. Il XXVIII congresso, svoltosi a Firenze tra l'11 e il 16 maggio 1949, vide la contrapposizione di tre mozioni: Per il Partito e per la classe, sostenuta da Jacometti, Lombardi e Santi; Per il socialismo, capeggiata da Romita e

${ }^{6}$ Cfr. FOA, Vittorio, Questo Novecento, Torino, Einaudi, 1996, p. 228.

57 Cfr. MATTERA, Paolo, Storia del PSI. 1892-1992, Roma, Carocci, 2010, pp. 150-151. 
infine la mozione di Sinistra, guidata da Nenni e Morandi (a cui stavolta si aggregò lo stesso Pertini) ${ }^{58}$.

In quei frangenti il clima di condizionamento del confronto bipolare si fece sentire in maniera pesante. La nascita della Nato, fermamente avversata da tutto il partito ${ }^{59}$, fu tra l'altro accompagnata da una nuova ventata di antiamericanismo cui fece da suggello la creazione a Parigi il 20 aprile del 1949 del Movimento dei Partigiani della Pace, di cui Nenni fu nominato vice-presidente ${ }^{60}$. Nel dibattito sull'adesione dell'Italia al nuovo organismo militare fu proprio Pertini a pronunciare in Senato un intervento particolarmente critico, definendolo «uno strumento di guerra», elemento di nuove divisioni all'interno dell'Europa in una prospettiva che finiva per essere meramente antisovietica, in quanto era chiaro che da una parte c'erano «le forze imperialistiche e plutocratiche, dall'altra le forze del lavoro» ${ }^{61}$.

Era questo il tono del dibattito che attraversò il congresso, da cui uscì vittoriosa la linea Nenni-Morandi: iniziava quella che è stata definita, in maniera suggestiva quanto efficace, la «seconda rifondazione del Psi» (la prima era stata quella del 1943-1944), caratterizzata da una riorganizzazione della burocrazia interna, più giovane e legata in maniera stretta al nuovo gruppo dirigente, pronta a diventare un formidabile strumento di stabilizzazione del consenso al nuovo corso $^{62}$. Centrale, in questa operazione, fu il completo allineamento al filosovietismo del Pci, cui l'intelligenza politica e organizzativa di Morandi aggiunse quegli elementi capaci di guidare un partito che doveva farsi trovare dalla parte giusta nel momento dello scontro inevitabile l'imperialismo capitalista e il mondo dei lavoratori63. Non erano ammessi sconti o distinguo, se non in talune posizioni originali ed eterogenee di un gruppo intellettuale

58 Sul congresso cfr. MATTERA, Paolo, Il partito inquieto, cit., pp. 155-157; SCIROCCO, Giovanni, Politique d'abord, cit., pp. 63-70.

59 In generale su questo tema ARDIA, Danilo, Il PSI e il Patto atlantico, Milano, Angeli, 1976.

60 Tutta l'azione di Nenni contro la ratifica del Patto Atlantico si trova ampiamente analizzata in TAMBURRANO, Giuseppe, Pietro Nenni, cit., pp. 255-259.

${ }^{61}$ Cfr. PERTINI, Sandro, Discorsi parlamentari 1945-1976, cit., pp. 38-42, seduta del Senato della Repubblica del 27 marzo 1949. Vedi anche PERTINI, Sandro, Anni di guerra fredda. Scritti e discorsi: 1947-1949, cit., pp. 278-282. Cfr. inoltre SCIROCCO, Giovanni, Politique d'abord, cit., p. 50.

${ }^{62}$ Per un'analisi innovativa della «seconda rifondazione» del Psi, attenta non solo alle strutture ma anche alle nuove forme della militanza del partito, vedi MATTERA, Paolo, «Dopo il 18 Aprile: la crisi e la "seconda rifondazione" del PSI», in Studi Storici, XLIII, 4/2002, pp. 11471179; ID., Il Partito inquieto, cit., pp. 168-195.

63 Sulla figura di Morandi cfr. AGOSTI, Aldo, Rodolfo Morandi. Il pensiero e l'azione politica, Roma-Bari, Laterza, 1971. Vedi anche le osservazioni di DEGL'INNOCENTI, Maurizio, Storia del Psi, cit., pp. 120-132 e SALVADORI, Massimo Luigi, La Sinistra nella storia italiana, cit., pp. 120-121. 
che si raccolse attorno a Panzieri64: non a caso a pagare per primi furono importanti dirigenti eterodossi come Basso ${ }^{65}$.

\section{Dopo la bufera. Pertini e il Psi negli anni del frontismo}

In questo schema fu sicuramente centrale l'uso strumentale del mito sovietico, potente strumento di compattamento interno che sanciva l'inutilità di ipotetiche terze vie in un momento in cui era la rigida logica dicotomica del bipolarismo a farla da padrone ${ }^{66}$. Un clima in cui fu totalmente coinvolto anche Pertini, che anni dopo avrebbe spiegato con queste parole tale atteggiamento:

Se non ti allineavi, non ti aiutava nessuno. E il Partito, ridotto in condizione tristissime dalla scissione e dalla sconfitta, aveva bisogno di aiuto. ${ }^{67}$

Non mancò da parte sua in quegli anni il ricorso a tutti gli artifici retorici utilizzati dalla propaganda filosovietica della sinistra italiana schierata su posizioni frontiste, come le lodi sperticate al sistema economico creato da Mosca, l'esaltazione del benessere egualitario vigente nella patria di Lenin, la valorizzazione dell'Urss come nazione che si batteva per la pace contro tutti i tentativi da parte del blocco occidentale di violare il clima di equilibrio nato dopo la fine della guerra, ad esempio in occasione della questione di Berlino ${ }^{68}$. Seguendo la prassi dei suoi compagni di partito, anche Pertini esaltava quindi i continui e inarrestabili progressi del mondo sovietico, contrapposti alle disuguaglianze del mondo americano. Quest'ultimo, in particolare, era

64 Si veda la recente e documentata ricerca di SCOTTI, Mariamargherita, Da sinistra. Intellettuali, Partito socialista italiano e organizzazione della cultura (1953-196o), Roma, Ediesse, 2011.

65 In proposito cfr. COLOZZA, Roberto, Lelio Basso. Una biografia politica (1948-1958), Ediesse, Roma 2010, p. 60 et seq. Su Basso nei primi anni della guerra fredda vedi ora l'ottimo lavoro di ROSSI, Emanuele, Democrazia come partecipazione. Lelio Basso e il PSI alle origini della Repubblica 1943-1947, Roma, Viella, 2011.

${ }^{66} \mathrm{Su}$ questi temi, in generale, ANDREUCCI, Franco, Falce e martello. Identità e linguaggi dei comunisti italiani fra stalinismo e guerra fredda, Bologna, Bononia University Press, 2005; MARIUZZO, Andrea, Divergenze parallele. Comunismo e anticomunismo alle origini del linguaggio politico dell'Italia repubblicana (1945-1953), Soveria Mannelli, Rubbettino, 2010; MATTERA, Paolo, Storia del Psi, cit., pp. 156-157; SCIROCCO, Giovanni, Politique d'abord, cit., pp. 98-110.

67 Cfr. TAMBURRANO, Giovanni, Lídentità socialista va difesa, in "Ciao Sandro», supplemento n. 2 di Argomenti Socialisti, Gennaio-Febbraio 1990, p. 81.

68 Cfr. PERTINI, Sandro, «Sull'orlo dell'abisso», in Lavoro Nuovo, 28 settembre 1948, ora in ID., Anni di guerra fredda. Scritti e discorsi: 1947-1949, cit., p. 256. Sui finanziamenti sovietici al Psi vedi ZASLAVSKY, Victor, Lo Stalinismo e la sinistra italiana, Milano, Mondadori, 2004. 
ritenuto responsabile di creare quell'atmosfera di odio con le sue mire di egemonia mondiale che avrebbero potuto portare ad una nuova, terribile guerra globale. Pertini denunciò in molti dei suoi articoli di quegli anni questo rischio; gli Stati Uniti volevano a suo avviso indebolire le conquiste del movimento operaio mondiale, mentre il popolo sovietico, dopo aver sconfitto eroicamente le armate di Hitler, non desiderava altro se non di vivere in tranquillità in una società dove finalmente i lavoratori avevano la possibilità di detenere il potere in una situazione di pace. In questo senso

l'Urss e questi popoli non possono volere la guerra, perché la loro opera, che si proietta nell'avvenire, ha bisogno di una pace sicura e duratura. 69

A suo avviso le forze conservatrici che dominavano la politica occidentale avevano esaltato l'Unione Sovietica quando serviva il suo impegno durante il secondo conflitto mondiale, ma ora non esitavano a combatterla perché si rendevano conto che erano in gioco i loro interessi e i loro privilegi ${ }^{70}$.

Questo spiega perché i suoi comizi di quel tempo, come quello di Roma del 13 marzo 1949, spesso si risolvessero in duri attacchi contro l'esecutivo De Gasperi, colpevole di essersi asservito alle potenze capitaliste e alle loro aspirazioni belliciste, contro le quali si sarebbero però sollevati «milioni di uomini e di donne per opporre alla guerra una insormontabile, paurosa barriera umana» ${ }^{71}$. Il Patto Atlantico veniva da lui giudicato come un vero e proprio strumento di guerra e di ulteriore divisione dell'Europa; in sostanza lo riteneva una nuova «Santa Alleanza» in funzione antisovietica, contro la nazione che aveva pagato il maggiore tributo in termine di vite umane durante la guerra al nazifascismo ${ }^{72}$.

Non mancò in questo senso la sua personale esaltazione del leader del Cremlino: in occasione del settantesimo compleanno di Stalin egli lo tratteggiò come

un rivoluzionario indomito, che si è battuto e si batte per il suo popolo e l'umanità tutta. [...] Compiuta la rivoluzione d'Ottobre, ha costruito il Socialismo nella sua patria e lo ha quindi irradiato nelle patrie altrui, sia nel cuore dell'Europa, sin

69 Cfr. PERTINI, Sandro, «Sull'orlo dell'abisso», in Lavoro Nuovo, 28 settembre 1948, ora in ID., Anni di guerra fredda. Scritti e discorsi: 1947-1949, cit., p. 256, pag. 257.

70 Cfr. PERTINI, Sandro, «Momento grave», in Lavoro Nuovo, 12 ottobre 1947, ora in ID., Anni di guerra fredda. Scritti e discorsi: 1947-1949, cit., pag. 114.

${ }^{71}$ Cfr. MATTERA, Paolo, Dopo il 18 Aprile: la crisi e la «seconda rifondazione» del PSI, cit., p. 1158.

72 Cfr. Atti Parlamentari, I Legislatura, Senato. Vol. V: Discussioni, 1948-1949, Seduta CLXXXIII, 27 marzo 1949, pp. 6562-6564, ora in PERTINI, Sandro, Anni di guerra fredda. Scritti e discorsi: 1947-1949, cit., pag. 280. 
nell'estremo Oriente, nell'immensa Cina. Tutti questi popoli che hanno conquistato la meta suprema e tutti gli altri che stanno per conquistarla guardano all'Urss come alla loro seconda patria e come alla roccaforte del Socialismo, contro cui si spezzerebbe ogni nuovo assalto delle forze imperialistiche.73

Bisogna tuttavia sottolineare come Pertini non sia certo da ascrivere alla categoria degli stalinisti ortodossi. Il suo richiamo era piuttosto dettato dall'idea di dover seguire quella che era la linea ufficiale del partito, anche se certo agiva su di lui il mito del capo della nazione che aveva tenuto in scacco i tedeschi a Stalingrado 74.

Ciò non toglie che certe sue affermazioni, specie quelle che fece dopo il canonico viaggio in Unione Sovietica, intrapreso in quegli anni da tanti esponenti della sinistra italiana e mondiale ${ }^{75}$, appaiano ingenue e superficiali, affini a quella visione acritica che i partiti della sinistra italiana avevano maturato nei primi anni del dopoguerra. Sacrificare l'umanitarismo socialista e la sua visione laica dell'azione politica all'esaltazione del culto della personalità di un uomo che pochi anni dopo sarebbe stato rinnegato dai suoi principali collaboratori fu infatti una decisione che ebbe un peso non indifferente nelle valutazioni politiche di migliaia di militanti del Psi e del $\mathrm{Pci}^{76}$. Certamente un uomo come Pertini non poteva non prestare interesse ad alcune realizzazioni concrete del mondo sovietico, a partire dall'ammirazione per la scomparsa di alcuni tratti di profonda arretratezza tipici della società russa che gli apparve quasi miracolosa77. O ancora la semplicistica constatazione dell'esistenza di un tasso quasi nullo di disoccupazione o di un alto livello di alfabetizzazione; tutte caratteristiche che lo portavano a giudicare il popolo russo come

un popolo tutto compatto, non tormentato da interni contrasti, intento ad una comune opera, proteso verso una meta unica. Dal più modesto lavoratore della terra o dell'officina, allo scienziato e allo scrittore celebri, dal semplice soldato dell'Armata Rossa al più alto dirigente dello Stato, tutti si sentono uniti da una

\footnotetext{
73 Cfr. PERTINI, Sandro, «Stalin», in Lavoro nuovo, 21 dicembre 1949, ora in ID., Scritti $e$ Discorsi di Sandro Pertini, cit., pp. 229-230.

74 Cfr. MATTERA, Paolo, Il Partito inquieto, cit., pp. 203-204.

75 Cfr. PERTINI, Sandro, «Di ritorno dall'URSS», in Avanti!, 19 marzo 1950, ora in ID., Scritti e Discorsi di Sandro Pertini, cit., pp. 247-249.

${ }^{76}$ Cfr. DE MARTINO, Francesco, Un'epoca del socialismo, cit., p. 142.

77 Cfr. DEGL'INNOCENTI, Maurizio, Introduzione, in CARETTI, Stefano, DEGL'INNOCENTI, Maurizio (a cura di), Sandro Pertini combattente per la libertà, Manduria-Roma, Lacaita, 1996, p. XXIV.
} 
stessa fede e da un compito comune. Per questo oggi in Russia si può dire sia stata politicamente e spiritualmente realizzata una vera unità nazionale..$^{7}$

Una visione certo edulcorata, non realistica e assolutamente antistorica, ma che corrispondeva interamente alla linea ufficiale del partito. Questo era il clima del Psi guidato da Nenni e Morandi; le critiche e i distinguo in quella fase non erano ancora ammessi. Gli unici che potevano provare a muovere dei rilievi erano i socialdemocratici, accusati per questo di essere portatori di un anticomunismo viscerale e privo di fondamenta79.

I socialisti come Pertini scelsero in sostanza il campo sovietico perché credevano che soltanto con la collaborazione e la guida dell'Urss avrebbero potuto realizzarsi la pace, la democrazia, il progresso in Italia e nel mondo ${ }^{80}$. Una scelta che era viziata da errori di fondo che il corso della storia avrebbe successivamente evidenziato, ma che s'iscriveva interamente nella stringente logica dicotomica della guerra fredda che il Psi aveva deciso di seguire.

Tanto più rilevante appare in questo contesto l'atteggiamento che i socialisti tennero in occasione della guerra di Corea, scoppiata il 25 giugno 1950.

Nenni e molti altri appoggiarono senza distinguo la causa dei coreani del Nord ${ }^{81}$, visti come la parte sana in grado di interpretare le richieste del popolo e l'ansia di giustizia sociale, a differenza di quelli del sud che volevano trasformare la penisola in un feudo statunitense ${ }^{82}$. Posizioni simili vennero espresse anche da Pertini, oramai diventato un «combattente» dei Partigiani della Pace ${ }^{83}$. Quel conflitto generò del resto la consapevolezza che ci si stesse avviando a grandi passi verso il terzo conflitto mondiale e che pertanto occorresse serrare i ranghi e rendere il partito sempre più compatto.

La situazione poté essere infranta solo da un evento di rottura come la scomparsa del dittatore georgiano nel marzo del 1953. Da quel momento si aprirono, seppur a piccoli passi, spiragli di un processo di distensione che fu uno degli elementi decisivi per il manifestarsi di nuove possibilità di manovra sul piano politico-istituzionale.

${ }^{78}$ Cfr. PERTINI, Sandro, «Di ritorno dall'URSS», in PERTINI, Sandro, Scritti e Discorsi di Sandro Pertini, cit., p. 248.

79 Cfr. VASSALLI, Giuliano, Introduzione, in PERTINI, Sandro, Scritti e Discorsi di Sandro Pertini, cit., p. XXVI.

${ }^{80}$ Cfr. SCIROCCO, Giovanni, Politique d'abord, cit., pp. 98-102.

${ }^{81}$ Cfr. GALLI, Giorgio, Storia del socialismo italiano, Roma-Bari, Laterza, 1980, p. 203.

82 Per l'atteggiamento di Nenni in seguito alla guerra di Corea, si rimanda a TAMBURRANO, Giuseppe, Pietro Nenni, Roma-Bari, Laterza, 1986, pp. 263-264.

83 Cfr. PERTINI, Sandro, «Le "Quinte colonne"», in Avanti! e Lavoro nuovo, 9 luglio 1950, ora in ID., Scritti e Discorsi di Sandro Pertini, cit., pp. 264-265. 
Insieme all'elezione di Eisenhower alla presidenza degli Stati Uniti ${ }^{84}$ e alla fine del conflitto coreano ${ }^{85}$, la scomparsa di Stalin segnò di fatto la fine di un'epoca. All'interno del dibattito del Psi Pertini vide l'occasione per rilanciare l'importanza strategica del suo partito, sola forza italiana a suo avviso capace di favorire il dialogo tra le forze politiche e il superamento delle barriere del periodo della guerra fredda grazie ad idee come la coesistenza pacifica. La sua era una visione che non teneva conto però delle scorie del frontismo e di quanto esse avessero condizionato in profondità il partito. Nuovi e drammatici eventi si sarebbero succeduti, a partire dai fatti tragici del 1956: solo da quel momento si sarebbe aperto nel concreto un nuovo corso del Psi verso la strategia della collaborazione al governo con la Dc nella formula del centro-sinistra. Anche in quel caso l'atteggiamento di Pertini, seppur ufficialmente allineato sulla posizione della maggioranza del partito, non fu tuttavia tenero con la svolta di Nenni e l'ipotesi di governo ${ }^{86}$. Gli eventi successivi, specie dopo il 1964 e in concomitanza con la sua ascesa alla Presidenza della Camera dei Deputati nel 1968, avrebbero definitivamente modificato le sue valutazioni circa gli assetti della politica internazionale, permettendogli infine di trovare un respiro e una profondità ben maggiore durante gli anni della Presidenza della Repubblica ${ }^{87}$.

84 Cfr. DEL PERO, Mario, Libertà e impero. Gli Stati Uniti e il mondo 1776-2006, Roma-Bari, Laterza, 2008, pp. 307-315.

85 Cfr. BONGIOVANNI, Bruno, Storia della guerra fredda, Roma-Bari, Laterza, 2001, pp. 9394; D'AGATA, Raffaele, Disfatta mondiale. Motivi ed effetti della guerra fredda, Roma, Odradek, 2007, pp. 65-70; GRAZIOSI, Andrea, L'Urss dal trionfo al degrado. Storia dell'Unione Sovietica, 1945- 1991, Bologna, Il Mulino, 2008, pp. 141-177; ROMERO, Federico, Storia della guerra fredda, cit., pp. 103-110.

86 In proposito SCROCCU, Gianluca, La passione di un socialista, cit., pp. 163-237.

87 Sulla presidenza Pertini vedi ora MAMMARELLA, Giuseppe, CACACE, Paolo, Il Quirinale. Storia politica e istituzionale da De Nicola a Napolitano, Roma-Bari, Laterza, 2011, pp. 171-198. Si veda anche RIDOLFI, Maurizio, Storia politica dell'Italia repubblicana, cit., pp. 96-99. 


\section{* L'autore}

Gianluca Scroccu, dottore di ricerca in Studi storici per l'età moderna e contemporanea presso l'Università di Firenze, è borsista presso il Dipartimento di Studi storici, geografici e artistici dell'Università di Cagliari. Si è occupato in particolare della storia politica e sociale dell'Italia repubblicana e delle vicende dei partiti della sinistra italiana, di storia di genere e delle figure di Sandro Pertini, Piero Gobetti e Giuseppe Garibaldi. Tra le sue pubblicazioni: Il PSI e la difficile ricerca del riformismo. Trasformazione degli equilibri interni e politica del dialogo negli anni di costruzione del centro-sinistra (1961-1962), in «Storia e futuro», 10, 2006, http://www.storiaefuturo.com/it/numero_10/articoli/1_psi-l-riformismo-trasformazionicostruzione-centro-sinistra 147.html; La passione di un socialista. Sandro Pertini e il PSI dalla Liberazione agli anni del centro-sinistra, Manduria-Bari-Roma, Lacaita, 2008; Il mondo visto da Torino. Asia e Africa nel lavoro politico e culturale di Piero Gobetti e Antonio Gramsci, in BALDUSSI, Annamaria, MANDUCHI, Patrizia (a cura di), Gramsci in Asia e Africa, Cagliari, Aipsa, 2010, pp. 51-73; Il partito al bivio. Il Psi dall'opposizione al governo (1953-1963), Roma, Carocci, 2011.

URL: < http://studistorici.com/progett/autori/\#Scroccu >

\section{Per citare questo articolo:}

SCROCCU, Gianluca, «Pacifismo, frontismo e autonomia: Pertini, il Psi e la "fase calda" della guerra fredda (19451950)», Diacronie. Studi di Storia Contemporanea: Quando la classe operaia andava in paradiso, 13/2/2012, URL:< http://www.studistorici.com/2012/02/13/scroccu_numero_9/ >

Diacronie Studi di Storia Contemporanea $\beta$ www.diacronie.it

Risorsa digitale indipendente a carattere storiografico. Uscita trimestrale. redazione.diacronie@hotmail.it

Comitato di redazione: Marco Abram - Giampaolo Amodei - Jacopo Bassi - Luca Bufarale - Alessandro Cattunar - Alice De Rensis Barbara Galimberti - Deborah Paci - Fausto Pietrancosta - Martina Sanna - Matteo Tomasoni - Luca Zuccolo

Diritti: gli articoli di Diacronie. Studi di Storia Contemporanea sono pubblicati sotto licenza Creative Commons 2.5 . Possono essere riprodotti a patto di non modificarne i contenuti e di non usarli per fini commerciali. La citazione di estratti è comunque sempre autorizzata, nei limiti previsti dalla legge. 